\title{
CALIDAD DE SERVICIO EN REDES DE TELECOMUNICACIONES
}

VALDEZ, Alberto D.; MIRANDA, Carlos A.; SCHLESINGER, Paola L.; CHIOZZA, Juan A.; MIRANDA, Carlos V.; GRELA, Abel A.(*)

\section{RESUMEN}

Las redes de telecomunicaciones que transportan información de diversa índole, en su gran mayoría en un formato digital independiente de su naturaleza, han crecido de una manera muy rápida en las dos últimas décadas. Este crecimiento trajo aparejado la utilización de velocidades muy elevadas, grandes anchos de banda y una variedad importante de medios físicos de transmisión. Si a esto le sumamos el desarrollo vertiginoso del procesamiento digital de la información y la multiplicidad de métodos, sistemas y software utilizados en pos de gran capacidad de manejo de datos de manera segura y confiable en el menor tiempo posible, no podemos dejar de hablar de la importancia de esquemas de Calidad de Servicio. Desde la primera comunicación telefónica analógica a las actuales basadas en señales digitales la tecnología evolucionó vertiginosamente, pero la calidad de servicio se mantuvo como un concepto de vital importancia. En el presente traba- jo se realiza una revisión integral basada en documentos relevantes sobre el tema.

Palabras claves: QoS, tráfico, procesamiento, prioridad, efectividad.

\section{INTRODUCCIÓN}

En el inicio las redes de telecomunicaciones se implementaban utilizando tecnologías basadas en técnicas de conmutación de circuitos. En este tipo de redes, al retener un circuito dedicado para cada comunicación, se cuenta con retardo mínimo y fijo, y también se asegura que no pueda existir congestión para las comunicaciones ya establecidas. Cualquier intento de conexión es bloqueado si la red no dispone de recursos suficientes. Las redes basadas en conmutación de circuitos, cuentan inherentemente con una alta calidad de servicio (Quality of Service QoS) ya que fueron diseñadas para soportar tráfico sensible a retardos y pérdidas de aplicaciones como voz y video [1].

(*) Facultad de Ciencias Exactas y Naturales y Agrimensura, Departamento de Ingeniería, UNNE, Corrientes CP3400, Argentina.dvaldez@exa.unne.edu.ar 
Las redes basadas en técnicas de conmutación de paquetes se diseñan utilizando algoritmos estadísticos. Estas redes son las más eficientes, y están adaptadas para brindar servicio a la mayoría de las aplicaciones, pero el retardo variable y la posible pérdida de información, pueden generar problemas al tráfico sensible a estos parámetros. En estas redes, en lugar de bloquear nuevos intentos de comunicaciones ante falta de recursos, las mismas se cursan, almacenando el tráfico excedente en buffers durante los picos de utilización. Esta técnica genera un retardo variable, correspondiente al tiempo que se almacenan los paquetes en los buffers. Como los buffers tienen un límite, durante periodos de congestión es posible que se descarten paquetes.

Las redes convergentes, que manejan múltiples servicios con una misma infraestructura, son la mejor opción hoy en día para los operadores, por su versatilidad y eficiencia con respecto a su costo, aunque es posible que no soporten aplicaciones específicas con la eficiencia que lo haría una red construida específicamente para dicha aplicación. Para aminorar este comportamiento, la aplicación de esquemas eficientes de QoS pasa a tomar un rol fundamental en redes convergentes, ya que la misma debe ser flexible y soportar muchos tipos de aplicaciones y servicios.

La función básica de un esquema de QoS es reconocer los diferentes flujos de tráfico provenientes de diferentes aplicaciones. Una vez que se ha reconocido el flujo, se puede proceder a especificar de qué forma se trata cada flujo de tráfico en la red.
De esta manera se puede priorizar un tipo sobre otro al utilizar los recursos de la red, como por ejemplo priorizar el tráfico sensible a retardos y pérdidas (tiempo real). El esquema de $\mathrm{QoS}$ de servicio permite controlar el acceso a los recursos disponibles.

Los principales parámetros con los que trabaja un esquema de QoS son:

Ancho de Banda (Bandwidtb): Define la capacidad de transferir información extremo a extremo. Cuando existe suficiente ancho de banda, no se encuentran problemas a nivel de calidad, ya que el esquema de QoS se aplica al existir congestión. De esta forma, incrementar el ancho de banda es una forma de resolver este problema.

Retardo (Delay): Define el retardo que existe en las comunicaciones entre los extremos. La causa del mismo es el retardo asociado a las líneas y tecnologías de transmisión, y el retardo de buffering asociado a las colas de tráfico en redes que usan multiplicación estadística.

Variación del Retardo (Jitter): Este parámetro indica los diferentes valores de retardo que pueden presentar los paquetes de una comunicación. Si bien un valor de retardo alto es indeseable, tener un valor variable es más disruptivo para las aplicaciones, ya que no es predecible y es difícil que las aplicaciones puedan ajustarse al mismo.

Pérdida (Loss): Este parámetro se refiere a la pérdida de paquetes de una comunicación. Para ciertas aplicaciones, como las que requieren transmisión en tiempo real, este parámetro puede ser muy disruptivo, ya que el costo de una retransmisión es por lo general más alto y disruptivo que la perdida 
de información. La retransmisión implica un incremento en el tiempo de transmisión normal. La pérdida de paquetes puede deberse a errores en los mismos, fallas en los equipos o congestión en la red (buffers excedidos).

En el presente trabajo se desarrollará de manera conceptual los temas centrales que involucran a la $\mathrm{QoS}$ en Redes de Telecomunicaciones, culminando con algunas conclusiones más bien generales dado, la diversidad y volumen de cada tópico, presentando algunas líneas de trabajo futuras. En particular se iniciará con las Recomendaciones de la Unión Internacional de Telecomunicaciones (UIT), continuando con la información de la Autoridad Federal de Tecnologías de la Información y las Comunicaciones (AFTIC), con la QoS en redes IP, distintas técnicas para abordarla, nuevos protocolos relacionados con la tecnología actual, modelos de análisis basados en el contexto, la QoS en redes móviles, las redes definidas por software y de próxima generación.

\section{DESARROLLO}

El esquema de QoS juega un rol fundamental en las redes de comunicaciones de hoy en día, e influye directamente en la percepción que los usuarios tienen del servicio de la red. Cada fabricante aplica un esquema de $\mathrm{QoS}$ en sus equipos, y al hacer un diseño es importante tener en cuenta la compatibilidad de los estándares.

El crecimiento de la capacidad de procesamiento de datos de los terminales móviles ha permitido la proliferación de aplicaciones multimedia intensivas en el uso de ancho de banda, como por ejemplo: streaming de video en alta definición (HD), multiconferencia y videoconferencias. Este enriquecimiento de los servicios ha permitido un aumento en el interés por parte de los usuarios móviles, generando un crecimiento de la demanda lo que, a su vez, impulsa a los operadores de dichas redes a que mejoren la infraestructura de sus redes, con el objetivo de dar capacidad ante este aumento de tráfico [2].

Hoy en día se utiliza ampliamente la expresión QoS, no sólo en el ámbito de las telecomunicaciones, del cual proviene, sino también en los servicios de banda ancha, inalámbricos y multimedios, que usan IP. En las redes y sistemas que se vienen diseñando se tiene cada vez más en cuenta la calidad de funcionamiento de extremo a extremo requerida por las aplicaciones de usuario; pero la expresión $\mathrm{QoS}$ casi nunca se define bien, o se la emplea con poca precisión o erróneamente. En lo que respecta a las definiciones posibles de calidad y QoS, ISO 8402 proporciona una definición de calidad mientras que la Rec. UIT-T E.800 suministra una definición de QoS: La definición de calidad general de ISO 8402 es: "el total de las características de una entidad que afectan su habilidad para satisfacer necesidades declaradas e implícitas" [3]. De igual manera, ISO 9000 define la calidad como "grado en el que un conjunto de características inherentes satisface los requisitos". Desde el punto de vista del usuario, la definición de ISO 8402 parece mejor. De todos modos, la QoS es clara- 
mente un subconjunto de la calidad global. Asimismo, la Rec. UIT-T E.800 define la QoS como "el efecto global de la calidad de funcionamiento de un servicio, que determina el grado de satisfacción de un usuario de dicho servicio". En la mayoría de las publicaciones, y normas, se utiliza la expresión "calidad de servicio" pero o no se la define, o se refiere a una de estas otras definiciones. Por ejemplo, en normas, informes y especificaciones de la industria no se define claramente la QoS, o se hace referencia a la Rec. UIT-T E.800.

Los criterios de calidad de un servi- cio de telecomunicación se pueden derivar a partir de la matriz que se presenta en la Figura 1. Se ha construido con mucha atención y se ha comprobado que es útil para identificar los criterios de QoS para los clientes antes de lanzar un nuevo servicio (ver ETR 003 del ETSI). La Comisión de Estudio de la FITCE (Federación de Ingenieros de Telecomunicación de la Comunidad Europea) concluyó que, según la granularidad de QoS que exija el servicio, era posible obtener hasta 43 criterios de QoS, de los que tan sólo 13 son importantes para el servicio de telefonía básica.

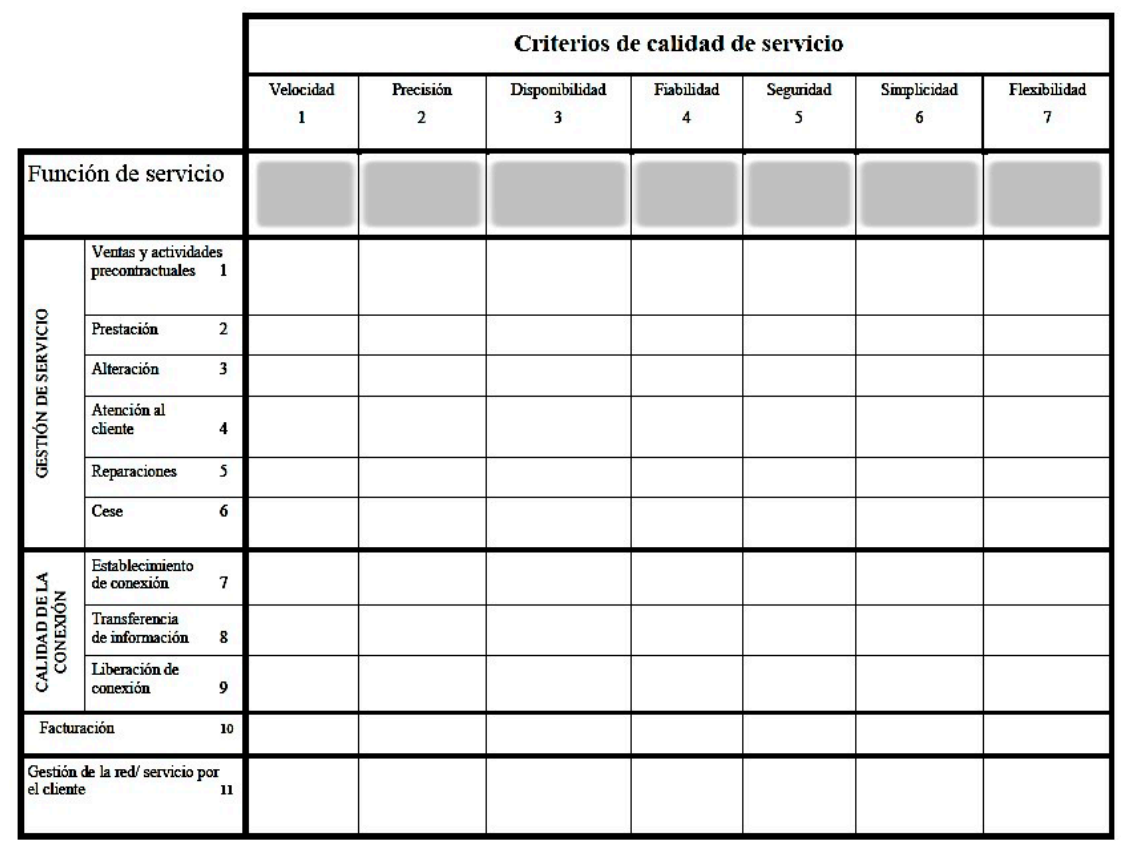

Figura 1. Matriz para facilitar la identificación de los criterios QoS para las comunicaciones [3].

La calidad de funcionamiento de la simples la calidad de funcionamiento del red contribuye a la QoS que experimenta acceso se separa normalmente de la caliel usuario/cliente. En la evaluación de dicha calidad se puede o no considerar la red como un todo. Por ejemplo, en las redes IP dad de funcionamiento de la red troncal, mientras que en el caso de Internet refleja con frecuencia las calidades combinadas de 
funcionamiento de red de varias redes autónomas.

En otras Recomendaciones UIT-T, como I.350 e Y.1540, se ha estudiado con más detalle la calidad de funcionamiento de la red y la parte de la QoS global que corresponde a interfaz de red y se comparan la QoS y la calidad de funcionamiento de la red. Desde el punto de vista del usuario, la Rec. UIT-T E.800 [4] sigue siendo la más significativa de las definiciones del UIT-T aunque, como se dijo, el marco de la Rec. UIT-T E. 800 que se propone es imprecisa en algunos aspectos y haya podido conducir a diversas interpretaciones. Es decir, aunque el marco de la Rec. UIT-T debería ser más concreto y centrarse en la aplicación, la definición básica de la QoS es válida.

En la matriz de definición de la QoS de la figura 1 se presentan criterios para juzgar la calidad de las funciones de comunicación que todo servicio debe soportar. Ahora bien, incluso esta matriz se puede analizar con perspectivas diferentes:

- Las necesidades de QoS del cliente;

- Las ofertas de QoS del proveedor de servicio (o QoS planificada/esperada);

- La QoS conseguida u ofrecida;

- La calificación de la QoS en las encuestas de cliente.

Para que un marco de QoS sea verdaderamente útil y lo suficientemente práctico para la industria, debe tener sentido en todas las perspectivas, como se ilustra en la Figura 2 y se define más adelante. Si bien la Figura 2 muestra las relaciones "descendentes" entre estos puntos de vista, no indica, por ejemplo, cómo el proveedor de servicio implementa la QoS; esto requiere métodos más detallados, con un enfoque "ascendentes", tema que no se aborda en esta Recomendación. El punto de esta Recomendación es que una sola definición y un solo marco de QoS pueden soportar todos los puntos de vista (Figura 2).

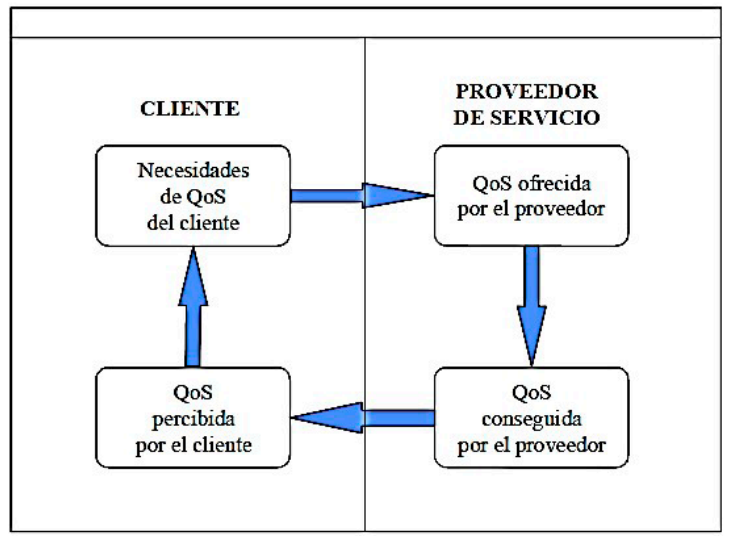

Figura 2. Puntos de vista sobre la QoS [3].

Las necesidades de QoS del cliente definen el nivel de calidad que se exige en un determinado servicio, y se pueden expresar en lenguaje corriente. Al cliente no le interesa saber cómo se presta el servicio ni los aspectos del diseño interno de la red, pues sólo le importa la calidad total del servicio de extremo a extremo. Desde el punto de vista del cliente, la QoS se expresa mediante parámetros que:

- Se ajustan a los efectos percibidos por el usuario, más que en sus causas dentro de la red.

- La forma de definirlo no depende de las hipótesis del diseño interno de la red.

- Considera todos los aspectos del servicio desde el punto de vista del cliente.

- El proveedor de servicio puede ga- 
rantizárselos al cliente, y hasta incluirlos en el contrato.

- Describen en términos independientes de la red e instauran un lenguaje común, que comprenden tanto el usuario como el proveedor de servicio.

La Rec. UIT-T G.1010, que trata las necesidades de QoS relativas a todas las aplicaciones de los usuarios finales, reviste interés especial, pues presenta explícitamente el punto de vista "necesidades del cliente", que puede servirle al proveedor de servicio para planificar su nivel de servicio de red.

La QoS ofrecida por el proveedor de servicio es una declaración del nivel de calidad que él espera ofrecer al cliente, y que se expresa mediante valores atribuidos a los parámetros. Esta forma de QoS es especialmente útil para la planificación y para los acuerdos de nivel de servicio. Cada servicio tendrá su propio conjunto de parámetros QoS (como en las clases de QoS de la Rec. UIT-T Y.1540 para los servicios IP). $\mathrm{El}$ proveedor de servicio puede expresar la QoS ofrecida en lenguaje corriente para el cliente, y en lenguaje técnico para su uso en la industria. Se puede utilizar la QoS ofrecida por el proveedor de servicio en los documentos de planificación para especificar los sistemas de medición y establecer las bases de los acuerdos de nivel de servicio. Por ejemplo, el proveedor puede manifestar, para informar al cliente, que ha planificado obtener una disponibilidad de servicio de telefonía básica de 99,95\% por año, con menos de 15 minutos de interrupción en cualquier momento, y no más de 3 inte- rrupciones en este periodo de un año. La QoS que consigue el proveedor de servicio es una declaración del nivel de calidad real alcanzado y entregado al cliente, y se expresa mediante valores asignados a parámetros, que deben ser idénticos a los especificados para la QoS ofrecida, de forma que se los pueda comparar para evaluar el nivel de calidad de funcionamiento logrado. Estos valores de calidad de funcionamiento se resumen para períodos específicos, por ejemplo el mes anterior. El proveedor de servicio puede, por ejemplo, declarar que la disponibilidad obtenida en el trimestre anterior fue de $99,95 \%$ con cinco interrupciones de servicio, una de las cuales duró 65 minutos. $\mathrm{La}$ industria, y a veces los reguladores, publican la QoS conseguida o entregada para información de los clientes.

La QoS percibida por los usuarios o clientes es una declaración en la que se expresa el nivel de calidad que ellos entienden haber experimentado, y que se expresa normalmente en función del grado de satisfacción y no en términos técnicos. Esta QoS se mide con encuestas a los clientes y sus comentarios sobre los niveles de servicio, y puede ser utilizada por el proveedor de servicio y el organismo de control para determinar la satisfacción del cliente en cuanto a la calidad de un servicio. Así, por ejemplo, un cliente puede decir que durante una cantidad inaceptable de ocasiones tuvo dificultad para realizar una llamada a través de la red y otorgar una calificación de 3 en una escala de 5 , donde 5 corresponde a un servicio excelente. Idealmente, debería haber una correspondencia uno a uno entre la 
QoS entregada y la percibida.

La Autoridad Federal de Tecnologías de la Información y las Comunicaciones de la República Argentina (AFTIC) pone a disposición del usuario a través del Portal Nacional de Usuarios de Telecomunicaciones “\#QUE NO SE CORTE” (www.quenosecorte.gob.ar) toda la información sobre la QoS de los servicios de telefonía e Internet prestados por las diferentes empresas de telecomunicaciones. Allí se encuentra la información suministrada por las empresas $\mathrm{y}$ por las mediciones efectuadas por el organismo estatal de control.

La QoS en el ámbito IP: El uso de redes y servicios basados en el IP, pone de manifiesto varios problemas, como la carencia de mecanismos normalizados, probados, robustos y escalables para:

- La atribución dinámica de recursos (como pérdida y retardo de paquetes) entre los segmentos de red.

- Asegurar que se alcanzan los objetivos de calidad de funcionamiento de red de extremo a extremo.

- La señalización perfecta de la QoS de extremo a extremo deseada a través de la red y las interfaces pares.

- La supervisión de la calidad de funcionamiento de las redes y los servicios basados en el IP conforme a los métodos utilizados en la planificación de redes y servicios y que tenga sentido desde el punto de vista de la experiencia del usuario.

- Un restablecimiento rápido y completo de la conectividad de capa IP tras interrupciones (o ataques) de envergadura en redes fuertemente sobrecargadas.
Es evidente que un enfoque coherente y global de la QoS es de gran utilidad para tratar estos aspectos en el momento oportuno.

Una de las primeras técnicas que surgió para tratar el problema de QoS, fue IntServ (Servicios Integrados), este modelo incluye dos clases para el control de QoS en Internet: servicio garantizado y servicio de carga controlada [5]. El primero asegura garantías de extremo a extremo a un flujo de datos. Aquí ningún flujo es descartado si el tráfico cumple con los parámetros establecidos. El servicio de carga controlada ofrece al usuario un envío con una QoS similar a la que obtendría en el caso de que la red estuviera libre, sin congestión. La señalización se realiza con el estándar RSVP (protocolo de reservación de recursos), donde un cliente solicita una determinada QoS para una aplicación determinada enviando un mensaje PATH al destino especificando las características que se deben cumplir. Los routers (encaminadores) intermedios a lo largo del camino envían el mensaje PATH a otros nodos hasta llegar al destino, que responde un mensaje RESV solicitando reserva de recursos para el flujo que será enviado. Los nodos intermedios pueden aceptar o no la petición; si la aceptan se reservan los recursos en caso contrario el proceso de señalización se termina.

Sin embargo IntServ presenta un problema de escalabilidad, debido a que la gran cantidad de información de control generada en la red incrementa proporcionalmente con el número de datos colapsando el núcleo de la networking (red) además de re- 
querir que el hardware soporte RSVP, control de admisión y clasificación.

Seguidamente surgió el protocolo Diffserv (Servicios Diferenciados) que clasifica el tráfico entrante en diferentes niveles de servicios, con el fin de aplicarle un comportamiento agregado a todos los datos de una determinada clase de servicio. Cada comportamiento se identifica con un único campo de servicio diferenciado (DS). Ese comportamiento se denomina comportamiento por salto PHB (per hop behavior). En el núcleo de la red, los paquetes son encaminados según el PHB asociado con su campo DS. Este campo DS tiene 8 bits de los cuales destina 6 para el campo denominado DSCP (Differentiated Services Code Point; punto de código de servicios diferenciados) para seleccionar el PHB que decide el comportamiento que se aplicará al tráfico que será encaminado a través del núcleo. Sin embargo a pesar de las bondades que ofrece, dada su escalabilidad y su fácil implementación capaz de ofrecer QoS de una forma diferenciada en una red congestionada, existe el problema de concentración de tráfico de alta prioridad en determinados puntos de la red, lo que puede afectar el tráfico de baja prioridad y causar degradación en el flujo de alta prioridad en los routers de borde.

Esta dinámica de migración a nuevas estructuras lógicas y físicas que funcionen mejor, surgen debido al crecimiento que ha tenido Internet en los últimos años y la variedad de aplicaciones que han ido apareciendo desde su creación; en tal sentido se hace necesario usar tecnologías de nueva ge- neración para el transporte de información a través de la súper autopista de las comunicaciones, ya que los usuarios finales cada vez demandan más y mejores servicios, los cuales tienen en su mayoría un alto contenido multimedia. Esto ha creado la necesidad de mejorar las características del servicio a fin de tener mayor eficiencia en el transporte a través de la red, pues este contenido en especial tiene una gran sensibilidad al retardo y también requiere un mayor ancho de banda. Parte de la solución para poder brindar un mejor desempeño de la red se ha dado por parte de las empresas prestadoras de servicios (Internet Service Provider ISP) aumentando las capacidades de sus enlaces, pero existe un límite físico y económico en el aumento de estos recursos. Esto ha obligado a que la industria y los investigadores enfoquen sus proyectos de investigación hacia el diseño, creación y evaluación de nuevos protocolos que resuelvan la problemática existente. Dentro de estas tecnologías de siguiente generación se encuentra MPLS (Multiprotocol Label Switching, Conmutación Multi Protocolo por Etiquetas) que surgió como una propuesta con el objetivo de unificar diferentes soluciones de conmutación multinivel, llamando la atención del mundo de las networking y definiéndose como una solución convergente de conectividad, la cual puede ser aplicada a cualquier estándar de capa de red, transformándose en una tecnología clave para la estrategia de comunicaciones de las empresas. MPLS ha incrementado las oportunidades de negocio para líneas dedicadas virtuales y servicios IP diferenciados, con una clasificación de 
tráfico que facilita el cobro de tarifas sobre dichos servicios [6]. Por tales motivos, en la actualidad varios proveedores de servicios de telecomunicaciones se han interesado en su implementación extendiendo la capacidad de negocio de las mismas al ofertar nuevas oportunidades en el manejo de ancho de banda e integración de servicios sobre la red. Posteriores investigaciones en MPLS probaron que una etiqueta podría ser mapeada a un color en el espectro soportando el procesamiento de datos no solo a nivel eléctrico sino también óptico, donde los paquetes podrían ser enlazados y reenviados directamente hacia una red óptica. Surge entonces el concepto de red inteligente que da como resultado la propuesta de generación de GMPLS (Generalized MPLS) [7], que es una versión extendida de MPLS, que no solamente realiza las labores de su predecesora, sino que además contiene elementos inteligentes especializados para descubrir vecinos, distribuir información de enlace, realizar administración de rutas y control centralizado. Esta tecnología ha podido convertirse en realidad gracias a los recientes avances en la construcción de dispositivos ópticos como multiplexores reconfigurables, conexiones ópticas cruzadas programables, que en combinación con la adopción de formatos modulación avanzados y de transductores permiten tomar una ventaja de la amplia capacidad en el ancho de banda provista por la multiplexación por división de longitud de onda (Wavelength Division Multiplexing WDM), además de eliminar el costo de la conversión eléctricaóptica por parte de los transductores, debi- do a que estos ya no serían usados en operaciones de intercambio, permitiéndole a la red ser transparente al tráfico de datos [8].

No obstante, la constante necesidad de buscar mejores soluciones a las exigencias impuestas por parte de los usuarios finales, ha hecho que la comunidad científica enfoque sus esfuerzos en la necesidad de disponer de una tecnología que conserve las mejores características de las tecnologías de transporte diseñadas para la conmutación de paquetes y lambdas dando origen al concepto de MPLS-TP (MPLS-Transport Profile; perfil de transporte) [9]. MPLSTP es una adaptación del MPLS para los requisitos del transporte. Es compatible totalmente con el MPLS, consiste básicamente en un conjunto de herramientas específicas para adecuarse a los requisitos de la red de transporte. Este entorno utiliza un subconjunto de las normas MPLS donde los elementos que no son requeridos en una red de transporte como el reenvío IP, retiro de etiquetas en los paquetes antes de llegar al router frontera, enrutamiento por múltiples rutas, son descartados y no soportados. Igualmente define las extensiones de los estándares MPLS ya existentes e introduce requisitos establecidos en las redes de transporte, incluyendo características de protección y un amplio conjunto de funciones de Operación y Mantenimiento (OAM) que ayudan en la rápida detección y localización de averías, resolución de problemas y supervisión del rendimiento. Estas características facilitan la transición a una red de transporte de paquetes orientada a la conexión, proporcionando una conmutación eficiente, al 
mismo tiempo que permite las operaciones de transferencia de alta calidad, a través de redes ópticas que aprovechen la tecnología MPLS.

Otra opción la constituye el Modelo de Análisis de Relaciones Paramétricas basado en el Contexto (Context-based Parametric Relationship Model CPRM). En base al paradigma actual y futura convergencia de las redes, en [10] se define un modelo para estudiar las relaciones paramétricas basado en el contexto, conocido más comúnmente como CPRM por sus siglas en ingles. Dicho modelo define la estructura de un sistema en base a un conjunto de parámetros y sus relaciones, un conjunto de operaciones que definen efectos sobre los parámetros dependientes, y una estructura de pesos que define la relevancia subjetiva y no subjetiva de los componentes del modelo. Por ejemplo, un administrador puede considerar subjetivamente que la confianza es un parámetro clave para la subsistencia del sistema de información. En ese caso, el parámetro confianza tendría un peso mayor en el sistema que otros parámetros menos relevantes dado el caso. A su vez, los mecanismos que implementen el valor de confianza podrían heredar la relevancia o peso de su parámetro padre, en este caso, el parámetro confianza. Estos valores subjetivos estarían sujetos a la variabilidad del contexto, de forma que en un momento dado, ya sea por las medidas de seguridad adoptadas o por el entorno donde está el individuo, su relevancia puede variar. Por ejemplo, en un entorno familiar bien definido, el parámetro confianza y los mecanismos estrechamente dependientes podrían relajar su relevancia de no existir otras dependencias que se lo impidan. Esto es así, porque en el contexto hogar el individuo podría asumir que la confianza viene dada por su ubicación. Aunque no tiene por qué ser así. Además, el modelo también contempla valores no subjetivos; destinados a definir el impacto o reacción en cadena que podría ocasionar una dependencia. Estos valores, se definen, en primer lugar, de forma aproximada en las dependencias del contexto general (GC, General Context), mientras que, una vez que los parámetros son instanciados, el peso es actualizado al contexto particular (PC, Particular Context).

Es muy importante el QoS ofrecida en redes móviles Ad Hoc (Mobile Ad-Hoc Networks - MANET-) que actualmente sólo ofrecen servicio best effort (mejor esfuerzo) [11]. Best Effort designa un tipo de servicio de red en el que no se puede garantizar que los datos lleguen a su destino, ni ofrecer al usuario un determinado QoS en sus comunicaciones. En una red de este tipo todos los usuarios reciben el mejor servicio posible en ese momento, lo que significa que obtendrán distintos anchos de banda y tiempos de respuesta en función del volumen de tráfico en la red. A diferencia de las redes inalámbricas tradicionales, las redes MANET son redes de comunicaciones formadas espontáneamente por un conjunto de dispositivos móviles, que son capaces de comunicarse entre sí, sin poseer una infraestructura ni una administración centralizada. Además, debido a que el alcance de transmisión de los dispositivos inalámbri- 
cos es limitado, pueden llegar a ser necesarios nodos intermedios para transferir datos de un nodo a otro a través de la red. Es por ello que los nodos cumplen funciones de fuentes, routers y destinos. La topología de las MANET es dinámica debido a que los nodos se mueven, se incorporan y abandonan la red continuamente; por esta razón, las condiciones de tráfico son altamente variables, haciendo extremadamente complicado dar buena QoS a las aplicaciones que la requieran.

Por otro lado, las variaciones en el canal de radio y las limitaciones de energía de los nodos pueden producir frecuentes cambios en la topología y en la conectividad. Consecuentemente, las MANET deben adaptarse dinámicamente para ser capaces de mantener las conexiones activas a pesar de estos cambios. Todas estas características hacen que las MANET sean Auto Creadas (Self Creating), Auto Organizadas (Self Organizing) y Auto Administradas (Self Administering) [12]. En una MANET la implementación de una solución de QoS permite un comportamiento determinístico de la misma, el cual se puede traducir en una priorización de cierto tipo de tráfico. Una adecuada implementación de un ambiente de provisión de QoS es necesaria para hacer más eficiente el uso y la asignación de los recursos de la red, mediante la identificación del tráfico con características críticas y restrictivas. Si bien la administración de QoS está prácticamente resuelta en redes fijas, las características de las redes MANET hacen necesario un nuevo estudio para afrontar este problema.
Las redes definidas por software (Software-Defined Network SDN) es uno de los principales temas de investigación, desarrollo y estandarización actuales [13]. Las tecnologías SDN flexibilizan el hardware y el software y permiten la posibilidad de asignación de recursos según la demanda para proporcionar cierta flexibilidad a la red. En la actualidad, OpenFlow es el protocolo SDN más utilizado.

El paradigma de servicios de red bajo demanda ("Network as a Service" o NaaS) [14] es la clave de las infraestructuras de redes futuras, una solución óptima a la falta de QoS en aplicaciones de tiempo real. El enfoque de $\mathrm{NaaS}$ permite una gestión dinámica y más escalable de los servicios de red.

Las aplicaciones en tiempo real demandan exigencias estrictas sobre el comportamiento de la red subyacente, pues requieren de ciertos parámetros fundamentales: baja latencia, mínima variación del retardo (jitter) y mínima pérdida de paquetes. La mayoría de los operadores de redes y proveedores de Internet sólo ofrecen servicios a medida de alta calidad para los clientes y servicios considerados "Premium". El común de los servicios utiliza políticas de mejor esfuerzo o Best-Effort, que llevan a servicios con comportamientos impredecibles y a la falta de fiabilidad en los proveedores de la red. Además, la carga de tráfico en la red varía con el tiempo debido al número de usuarios simultáneos que la utilizan, creando un grave impacto sobre la elasticidad y escalabilidad de la red. Por lo tanto, el reto se encuentra en hacer que la nueva tecnología funcione de una manera que cumpla 
con los requisitos propios en tiempo real y que proporcione a la red de la flexibilidad necesaria para las aplicaciones. E1 SDNApp Pathfinder [15] es una aplicación externa diseñada para funcionar junto con un controlador SDN y se basa en sus técnicas específicas para proporcionar y mantener la QoS de extremo a extremo. Se hace uso de mecanismos de cola para mapear un flujo con ciertos parámetros a una cola específica en un puerto. Así, los principales objetivos del proyecto son: proveer QoS extremo a extremo bajo demanda sobre una red SDN para solicitudes QoS de aplicaciones en tiempo real; buscar caminos factibles que cumplan los requerimientos de QoS y seleccionar la ruta más óptima para asegurar la calidad requerida; reconfigurar dinámicamente la red para adaptarse a los cambios y requisitos de la aplicación; monitorizar el estado de la red mediante la obtención de datos estadísticos de los dispositivos de red y calcular rutas alternativas para el tráfico en caso de detectar violaciones del acuerdo QoS y crear un conjunto de aplicaciones SDNApp diseñadas para trabajar junto con un controlador de SDN, como el controlador Floodlight, o integradas como recursos en el NCL (Network Control Layer, Capa de Control de Red). El cálculo de Pathfinder consiste en una heurística que analiza y funciona a través de una estructura gráfica de topologías de red. Los mecanismos de consultas de estado paralelos y el propio controlador recuperan las estadísticas de cada switch de red proporcionando la estructura gráfica de la topología. Utilizando esta información, se ha desarrolla- do un método para determinar el camino óptimo o adecuado utilizando estadísticas del estado de la red y también algoritmos de cálculo de rutas que siguen las siguientes técnicas codiciosas: reducción del grafo de la red QoS y ruta de máximo peso/longitud. SDN Pathfinder es capaz de buscar y seleccionar el camino adecuado para garantizar una QoS mínima para el tráfico de datos necesario entre las aplicaciones en tiempo real, objetivo logrado en conjunción con módulos SDN paralelos que requiere la aplicación SDN para lograr su principal función de suministro y control. El algoritmo de Pathfinder hace uso de la capacidad de programación de la red para volverla más eficiente y eficaz según las necesidades de los clientes. Un algoritmo de enrutamiento de QoS debe reducir al mínimo la tasa de rechazo de las solicitudes, mientras se mantiene o mejora el rendimiento y/o calidad global de la red (es decir, aquellos parámetros comunes de la QoS, como el retardo, la tasa de pérdida o de alto rendimiento). E1 principal objetivo de Pathfinder es ofrecer QoS extremo a extremo (e2e) en una red definida por software, primero buscando aquellos caminos factibles, luego seleccionando el camino más adecuado para poder enrutar entonces los flujos de paquetes a través de los circuitos creados sobre los enlaces calculados cumpliendo con las demandas de los clientes, mientras se mantiene estable el rendimiento general de la red y la calidad, tratando de minimizar la tasa de rechazo de las solicitudes.

Existe un proyecto MIRA desarrollado por investigadores españoles. El mismo 
está basado en el desarrollo de una plataforma avanzada de supervisión y control de tráfico para redes académicas y de investigación. El proyecto MIRA es fruto de la evolución de distintos proyectos orientados al análisis y caracterización de tráfico en RedIRIS [16]. En proyectos anteriores del mismo grupo, se desarrollaron herramientas para la caracterización del tráfico basado en el análisis de cabeceras TCP/UDP/IP y de aplicación (CASTBA2), de análisis de contenidos, así como de los orígenes y destinos del tráfico con gran granularidad (MEHARI3). En este proyecto se integran distintos métodos de análisis de tráfico, basados en parámetros derivados de las cabeceras de los paquetes a distintos niveles (ATM, AAL5, IP, Transporte y Aplicación), así como parámetros derivados del análisis de contenidos. Se desarrollaron nuevos mecanismos que permitirán analizar el comportamiento de las redes con topología compleja y prever sus tendencias. Se amplían también los heurísticos para la caracterización del tráfico, al mismo tiempo que se ofrecen resultados cruzados entre módulos de análisis de los proyectos anteriores. La parte software se ha estructurado como una jerarquía de módulos a cuatro niveles. Dentro de cada jerarquía se realizan funciones del mismo tipo, pero cada módulo está especializado en una tarea determinada. La interfaz entre los distintos módulos sigue una misma especificación que permite encadenar módulos de diferentes jerarquías y combinarlos para obtener resultados más complejos mediante un lenguaje propio de filtrado de datos. Finalmente, también se han desarrollado herramientas de tratamiento gráfico, tanto para los resultados (generación y visualización de gráficas) como para el control de los procesos (GUI, Graphic User Interface). El resultado final es una arquitectura de procesos de análisis de tráfico abierto, que integra, además de los procesos de captura y análisis, todas las funcionalidades relacionadas con el tratamiento masivo de datos y la generación y consulta de resultados.

Las normas para la QoS en Redes de Siguiente Generación (Next Generation Networking, NGN) permiten analizar un tipo de redes basadas en paquetes que realizan la transmisión de datos a través de infraestructuras convergentes. La norma ETSI TS 185001 [17] provee un conjunto de conceptos QoS genéricos para NGN, un modelo de marco teórico y describe los requerimientos para la entrega de $\mathrm{QoS}$ en NGN. De acuerdo con esta norma, las redes NGN pueden soportar el desarrollo de los siguientes modelos. QoS garantizada: es el servicio de entrega de tráfico con límites numéricos en algunos o todos los parámetros. QoS relativa: es el servicio de entrega de tráfico sin límites absolutos en la administración de ancho de banda, retardo de paquetes o pérdida de paquetes. También existen dos tipos de definiciones de QoS en esta norma: QoS extremo a extremo - end to end QoS ó QoS en el portador (red de transmisión y distribución).

$$
\text { Los requerimientos de QoS en NGN }
$$
es ser capaz de soportar un amplio rango de servicios de QoS habilitados. Para ofre- 
cer estos servicios es necesario definir QoS en los mecanismos de control y en la arquitectura de control.

Además, definir QoS en el control de la señalización. Debe soportar diferentes tipos de Codecs; además, soportará negociación de Codecs entre entidades NGN (terminales, elementos de red). Debe tener en cuenta diferentes mecanismos de control QoS correspondientes a diferentes tecnologías y posiblemente diferentes modelos de negocios. La arquitectura QoS de NGN debe ser capaz de administrar diferentes tipos de redes de acceso (ejemplo: xDSL, redes de acceso $3 \mathrm{GPP}$, etc.). El control de señalización NGN QoS debe estar basado en protocolos ya definidos o protocolos bajo desarrollo.

Los parámetros comunes entre las normas Y.1541 [18] y ETSI TS 185001 son los siguientes: IPTD (IP Packet Transfer Delay, Retardo de Transferencia de Paquete) hace referencia al tiempo que tarda el paquete en pasar por un componente de la red. Esta entidad puede ser un host, un enrutador o una sección de red. Este parámetro es uno de los parámetros principales y críticos para todas las aplicaciones que utilicen NGN. IPDV (IP Packet Delay Variation, Variación del Retardo del Paquete) hace referencia al jitter o al tiempo esperado de llegada de cada paquete. IPLR (IP Packet Loss Ratio, relación de Pérdida del Paquete) hace referencia a la razón de pérdida de paquetes, la cual se obtiene entre el total de paquetes perdidos sobre el total de paquetes transmitidos en un flujo de datos determinado. IPER (IP Packet Error Ratio, Tasa de
Paquetes con Errores) hace referencia a la tasa de paquetes con errores, la cual se obtiene entre el total de paquetes con errores sobre el total de paquetes transmitidos en un flujo de datos determinado. En general es posible afirmar que el concepto de QoS en redes NGN abarca una gran cantidad de diferencias con las redes tradicionales dado la flexibilidad de servicios y tipos de tráfico. Entre las diferentes normas existentes que permiten medir QoS, algunos parámetros comunes son el retardo de paquetes, la variación del retardo, la pérdida de paquetes y la cantidad de paquetes con errores.

\section{CONCLUSIONES}

La calidad de servicio, QoS, en los sistemas actuales de telecomunicaciones es un tema de gran complejidad, objeto de estudio permanente. El desarrollo de nuevas tecnologías de comunicación sobre las teorías existentes, la utilización de nuevos aspectos de la teoría de la información, la accesibilidad a nuevos materiales de excelentes características técnicas y costos reducidos, el avance sostenido de los sistemas electrónicos funcionando en bandas de mayores frecuencias y velocidades de datos han dado un impulso cuasi exponencial a las comunicaciones electrónicas. La flexibilidad, la capacidad, la adaptabilidad de esquemas de procesamiento de señales digitales y la complejidad resultante en las actuales redes de telecomunicaciones, hacen indispensable contar con métodos que aseguren una adecuada calidad de servicio. Lejos quedó la Recomendación UIT-T G1000 en lo que a tecnología se refiere pero muy actual en lo 
que hace a los conceptos de Calidad de Servicio definidos en la misma, los cuales cobran importancia sustancial en el manejo de la información por medios electrónicos digitales. Sin embargo la UIT a través de sus Recomendaciones a lo largo de estos años fue marcando un camino en ésta búsqueda. El desarrollo de las redes móviles en sus sucesivas generaciones fue orientado a buscar aumentar la velocidad de transferencia a la cual puede acceder el usuario por medio de un enlace por radio propagación de manera más eficiente y segura, lo cual trae aparejado la necesidad de trabajar en la Calidad de Servicio de una manera más detallada. Otro punto importante de desarrollo en la actualidad lo constituye la fibra óptica como medio principal de desarrollo tecnológico con capacidades muy grandes. El desarrollo de las redes NGN trajo aparejado un conjunto de conceptos sobre la Calidad de Servicio emergentes de la complejidad y variedad de infraestructuras convergentes.

\section{IV.LÍNEAS FUTURAS DE TRA- BAJO}

Las líneas de investigación actuales a nivel mundial apuntan al objetivo fundamental de transmitir la información de la manera más rápida, segura y eficiente. La complejidad creciente de los sistemas de información sobre redes de telecomunicaciones genera múltiples líneas de investigación a nivel académico y de empresas. Los diferentes grupos de investigación se encuentran realizando avances en cuestiones puntuales sin concretarse aún programas de investigación colaborativa. Uno de los sistemas interesantes para trabajar en mejorar la QoS son aquellos que se realizan en tiempo real tal como la transmisión de video. Las modernas redes LTE que se están implementando en la República Argentina orientadas a las altas velocidades de transmisión, haciendo hincapié en video de tiempo real, involucran la necesidad imperiosa de que la QoS sea la adecuada al servicio ofrecido. El servicio de conectividad IP proporcionado por las redes LTE debe soportar QoS para lograr que los paquetes de datos de una determinada conexión sean tratados de modo diferenciado. Esto permitirá que las redes LTE soporten múltiples servicios con diferentes requisitos. E1 estudio de la gestión de QoS en redes LTE es un tema que puede ser de interés. Es de esperarse que en el futuro se puedan aunar esfuerzos en pos de lograr un enfoque global de la Calidad de Servicio en Redes de Telecomunicaciones. 


\section{REFERENCIAS}

[1] Auben. Calidad de Servicio.

http://www.auben.net/index.php 27 de mayo de 2015.

[2] Segura Villalobos, C. D. "Diseño e Implementación de una Metodología para la Medición de QoS/QoE en Servicios OTT Montados sobre una Plataforma LTE/IMS”. 2012.

[3] UIT. Recomendación UIT-T G1000. "Serie G: Sistemas y Medios de Transmisión, Sistemas y Redes Digitales. Calidad de servicio y de transmisión”. 2001.

[4] UIT. Recomendación UIT E.800 Serie E: Explotación General de la Red, Servicio Telefónico, Explotación del Servicio y Factores Humanos. Calidad de los servicios de telecomunicación: conceptos, modelos, objetivos, planificación de la seguridad de funcionamiento - Términos y definiciones relativos a la calidad de los servicios de telecomunicación. 2008.

[5] García, Rocío C., Salcedo, Octavio J., López, Danilo A. y Pedraza, Luis F. "Evaluación de la Calidad del Servicio para Voz sobre Protocolo de Internet (VoIP) en Redes WIMAX sobre Ambientes IP/MPLS”. Información Tecnológica Vol. 25(2), 24-38. Doi: 10.4067/S0718-

07642014000200004. 2014.

[6] El-Alfy, E., S. Mujahid., S. Selim, "A Pareto-based hybrid multiobjective evolutionary approach for constrained multipath traffic engineering optimization in MPLS/GMPLS networks", J. Network and Computer Applications 36 (4), 1196-1207. 2013.

[7] Cisco. Cisco Segmented Generalized Multiprotocol Label Switching. www.cisco.com. 8 de Julio 2015.

[8] Ayan Banerjee, John Drake, Jonathan P. Lang, and Brad Turner, Calient Networks Kireeti Kompella, Juniper Networks Yakov Rekhter, "Generalized Multiprotocol LabelSwitching: An Overview of Routing and Management Enhancements". Cisco Systems. IEEE Communications Magazine. Enero 2001

[9] Vilalta, R., Muñoz R., Casellas, H., Martinez R., J.
Wilchez J., "GMPLS-enabled MPLS/PWE3 node with integrated 10 Gbps tunable DWDM transponders: design and experimental evaluation", Original Research Article Computer Networks, Volume 56, Issue 13, 3123-3135. 2012

[10] Nieto A., López J., "Herramienta para la Compensación de Parámetros de QoS y Seguridad". RECSI 2014, Alicante, 2-5 septiembre 2014.

[11] Kui Wu. Janelle Harms. QoS Support in Mobile Ad Hoc Networks.University of Alberta. 2005.

[12] Barbeau y Kranakis. "Principles of Ad-Hoc Networking”. John Wiley and Sons. 2007.

[13] Thomas D. Nadeau, Ken Gray "SDN: Software Defined Networks". O’Reilly. 2013.

[14] I. Bueno, J. I. Aznar, E. Escalona, J. Ferrer, J.A. García- Espín. "An OpenNaaS based SDN Framework for Dynamic QoS control”, Future Networks and Services (SDN4FNS), 2013 IEEE SDN for. IEEE, 2013.

[15] Daniel Guija Alcaraz. "Gestión y control de Calidad de Servicio de redes SDN en tiempo real- Pathfinder, una SDNApp para el aprovisionamiento de Calidad de Servicio". Escola Politècnica Superior d'Enginyeria de Vilanova i la Geltrú, UPC - EPSEVG Vilanova i la Geltrú, Barcelona, España. 2014.

[16] Veciana Carles, Solé Pareta Josep, Sales Sergi, Jordi Domingo. "MIRA: Software para el análisis de tráfico IP sobre ATM“. Universitat Politècnica de Catalunya (UPC). 2013.

[17] ETSI TS 185001 V1.1.1. "Telecommunication and Internet converged Services and Protocols for Advanced Networking (TISPAN); Next Generation Network (NGN); Quality of Service (QoS) Framework and Requirements". 2005.

[18] UIT. Recommendation ITU-T Y.1541. "Series Y: Global Information Infrastructure, Internet Protocol Aspects andNext-Generation Networks. Internet protocol aspects - Quality of service and network performance”. 2011. 